\title{
A note on functional equations connected with the Cauchy mean value theorem
}

\author{
RADOSEAW EUKASIK(D)
}

Abstract. The aim of this paper is to describe the solution $(f, g)$ of the equation

$$
[f(x)-f(y)] g^{\prime}(\alpha x+(1-\alpha) y)=[g(x)-g(y)] f^{\prime}(\alpha x+(1-\alpha) y), x, y \in I,
$$

where $I \subset \mathbb{R}$ is an open interval, $f, g: I \rightarrow \mathbb{R}$ are differentiable, $\alpha$ is a fixed number from $(0,1)$.

Mathematics Subject Classification. 39B22.

Keywords. Functional equation, Mean value theorem, Linearly dependent functions.

\section{Introduction}

Throughout this paper $I$ is an open interval, $\alpha \in\left(0, \frac{1}{2}\right]$ (we can obtain the whole interval $(0,1)$ because the role of $\alpha$ and $1-\alpha$ is symmetric in (1)). For a differentiable function $f: I \rightarrow \mathbb{R}$ we define a set $U_{f}:=\left\{x \in I: f^{\prime}(x) \neq 0\right\}$. In view of the Darboux property of $f^{\prime}$ we can write $U_{f}$ as a sum of pairwise disjoint open intervals (we denote this family of open intervals by $\mathcal{A}_{f}$ ).

We would like to present solutions of the following functional equation

$$
[f(x)-f(y)] g^{\prime}(\alpha x+(1-\alpha) y)=[g(x)-g(y)] f^{\prime}(\alpha x+(1-\alpha) y), x, y \in I,
$$

where $f, g: I \rightarrow \mathbb{R}$ are differentiable.

This equation was solved by Balogh et al. [2] for three times differentiable functions on $\mathbb{R}$. For the case of the Lagrange MVT $(g=\mathrm{id})$ with $\alpha=\frac{1}{2}$ this problem was considered by Haruki [3] and Aczél [1]. The generalization of (1) corresponds also to an open problem posed by Sahoo and Riedel [6]. 


\section{Auxiliary results}

We divide our considerations into two cases $\alpha \neq \frac{1}{2}$ or $\alpha=\frac{1}{2}$. We start with two lemmas which we use in both cases.

Lemma 1. Let $f, g: I \rightarrow \mathbb{R}$ be differentiable functions such that (1) holds. Then for each $x \in\left((1-\alpha) \inf I+\alpha \inf U_{f},(1-\alpha) \sup I+\alpha \sup U_{f}\right)$ such that $\exists_{\varepsilon>0}\left((x-\varepsilon, x] \subset I \backslash U_{f}\right.$ or $\left.[x, x+\varepsilon) \subset I \backslash U_{f}\right)$ we have $g^{\prime}(x)=0$.

Proof. Let $J \subset I \backslash U_{f}$ be a maximal closed subinterval of $I \backslash U_{f}, a=\inf J$, $b=\sup J, a<b$, which means that either $J=[a, b]$ and $a, b \in \operatorname{cl} U_{f}$ or $J=\left(\inf I, \inf U_{f}\right]$ or $J=\left[\sup U_{f}, \sup I\right)$. We have the following cases:

(a) $\exists_{I_{1} \in \mathcal{A}_{f}} \sup I_{1}=a$.

Let $z \in[a, \alpha a+(1-\alpha) b)$, then there exists $x \in I_{1}$ such that $a-x<$ $\frac{\alpha a+(1-\alpha) b-z}{\alpha}$. Let $y=b-\frac{\alpha x+(1-\alpha) b-z}{1-\alpha}$. Then $z=\alpha x+(1-\alpha) y$. Since

$$
\begin{gathered}
(1-\alpha)(b-y)=\alpha x+(1-\alpha) b-z=\alpha(x-a)+\alpha a+(1-\alpha) b-z \\
>-(\alpha a+(1-\alpha) b-z)+\alpha a+(1-\alpha) b-z=0, \\
y=\frac{z-\alpha x}{1-\alpha}=\frac{z-\alpha a+\alpha(a-x)}{1-\alpha}>\frac{z-\alpha a}{1-\alpha} \geq \frac{a-\alpha a}{1-\alpha}=a,
\end{gathered}
$$

we have $y \in J$. Hence

$$
0=[g(x)-g(y)] f^{\prime}(z)=[f(x)-f(y)] g^{\prime}(z)=[f(x)-f(a)] g^{\prime}(z) .
$$

Since $\left.f^{\prime}\right|_{I_{1}} \neq 0$, we have $f(x) \neq f(a)$ and $g^{\prime}(z)=0$.

(b) $\exists_{\left(I_{n}\right)_{n \in \mathbb{N}} \subset \mathcal{A}_{f}}\left(\forall_{n \in \mathbb{N}} \sup I_{n} \leq \inf I_{n+1}\right) \wedge \lim _{n \rightarrow \infty} \sup I_{n}=a$. Let $z \in[a, \alpha a+$ $(1-\alpha) b)$, then there exists $n \in \mathbb{N}$ such that $a-\inf I_{n}<\frac{\alpha a+(1-\alpha) b-z}{\alpha}$. Let $x \in I_{n}$ be such that $f(x) \neq f(a), y=b-\frac{\alpha x+(1-\alpha) b-z}{1-\alpha}$. Then $z=\alpha x+(1-\alpha) y$. Similarly as in the previous case we have $y \in J$. Hence (2) holds and $g^{\prime}(z)=0$.

(c) $\exists_{I_{1} \in \mathcal{A}_{f}}$ inf $I_{1}=b$. Let $z \in(\alpha b+(1-\alpha) a, b]$, then there exists $x \in I_{1}$ such that $x-b<\frac{z-\alpha b+(1-\alpha) a}{\alpha}$. Let $y=a+\frac{z-\alpha x-(1-\alpha) a}{1-\alpha}$. Then $z=$ $\alpha x+(1-\alpha) y$. Since

$$
\begin{gathered}
(1-\alpha)(y-a)=z-\alpha x-(1-\alpha) a=z+\alpha(b-x)-\alpha b-(1-\alpha) a \\
>z-(z-\alpha b-(1-\alpha) a)-\alpha b-(1-\alpha) a=0 \\
y=\frac{z-\alpha x}{1-\alpha}=\frac{z-\alpha b+\alpha(b-x)}{1-\alpha}<\frac{z-\alpha b}{1-\alpha} \leq \frac{b-\alpha b}{1-\alpha}=b
\end{gathered}
$$

we have $y \in J$. Hence (2) holds. Since $\left.f^{\prime}\right|_{I_{1}} \neq 0$, we have $f(x) \neq f(b)$ and $g^{\prime}(z)=0$.

(d) $\exists_{\left(I_{n}\right)_{n \in \mathbb{N}} \subset \mathcal{A}_{f}}\left(\forall_{n \in \mathbb{N}} \inf I_{n} \geq \sup I_{n+1}\right) \wedge \lim _{n \rightarrow \infty} \inf I_{n}=b$. Let $z \in(\alpha b+$ $(1-\alpha) a, b]$, then there exists $n \in \mathbb{N}$ such that $\sup I_{n}-b<\frac{z-\alpha b+(1-\alpha) a}{\alpha}$. 
Let $x \in I_{n}$ be such that $f(x) \neq f(b), y=a+\frac{z-\alpha x-(1-\alpha) a}{1-\alpha}$. Then $z=\alpha x+(1-\alpha) y$. Similarly as in the previous case we have $y \in J$. Hence (2) holds and $g^{\prime}(z)=0$.

Since

$$
\alpha b+(1-\alpha) a=a+\alpha(b-a) \leq \frac{a+b}{2} \leq b-\alpha(b-a)=\alpha a+(1-\alpha) b,
$$

for $J \subset\left(\inf U_{f}, \sup U_{f}\right)$ we have $g^{\prime}(x)=0$ for $x \in J$.

If $J=\left(\inf I, \inf U_{f}\right]$, then $g^{\prime}(x)=0$ for $x \in\left((1-\alpha) \inf I+\alpha \inf U_{f}, \inf U_{f}\right]$.

And finally, if $J=\left[\sup U_{f}, \sup I\right)$, then $g^{\prime}(x)=0$ for $x \in\left[\sup U_{f},(1-\right.$ $\left.\alpha) \sup I+\alpha \sup U_{f}\right)$.

Lemma 2. Let $f, g: I \rightarrow \mathbb{R}$ be differentiable and satisfy Eq. (1). Assume that $\{1, f, g\}$ are linearly dependent on each $J \in \mathcal{A}_{f}$ and $\inf I=-\infty$ or $\sup I=$ $+\infty$. Then $\{1, f, g\}$ are linearly dependent on $I$.

Proof. It is easy to see that if $f$ is constant, then for every $g$ Eq. (1) holds.

Assume that $f$ is non-constant and $\sup I=+\infty$.

Let $I_{1}, I_{2} \in \mathcal{A}_{f}$ satisfy $\sup I_{1} \leq \inf I_{2}$. There exist $c_{1}, c_{2}, d_{1}, d_{2} \in \mathbb{R}$ such that $g(x)=c_{1} f(x)+d_{1}$ for $x \in I_{1}$ and $g(x)=c_{2} f(x)+d_{2}$ for $x \in I_{2}$. For each $x \in I_{1}$ and $z \in I_{2}$ we define $y_{x, z}=\frac{z-\alpha x}{1-\alpha} \in I$. Then $z=\alpha x+(1-\alpha) y_{x, z}$. We have

$$
\begin{aligned}
{[f(x)} & \left.-f\left(y_{x, z}\right)\right] c_{2} f^{\prime}(z)=\left[f(x)-f\left(y_{x, z}\right)\right] g^{\prime}(z) \\
& =\left[g(x)-g\left(y_{x, z}\right)\right] f^{\prime}(z)=\left[c_{1} f(x)+d_{1}-g\left(y_{x, z}\right)\right] f^{\prime}(z), x \in I_{1}, \quad z \in I_{2} .
\end{aligned}
$$

Hence

$$
\left(c_{1}-c_{2}\right) f(x) f^{\prime}(z)=\left[g\left(y_{x, z}\right)-c_{2} f\left(y_{x, z}\right)-d_{1}\right] f^{\prime}(z), x \in I_{1}, \quad z \in I_{2},
$$

and since $f^{\prime}(z) \neq 0$, we obtain

$$
\left(c_{1}-c_{2}\right) f(x)=g\left(\frac{z-\alpha x}{1-\alpha}\right)-c_{2} f\left(\frac{z-\alpha x}{1-\alpha}\right)-d_{1}, x \in I_{1}, z \in I_{2} .
$$

Using the differentiation of the above equation with respect to $z$ we obtain that RHS of (3) is constant and also LHS of (3) is constant. The function $\left.f\right|_{I_{1}}$ is injective so we get $c_{1}=c_{2}$.

This shows us that there exists $c \in \mathbb{R}$ such that

$$
\forall \forall_{J \in \mathcal{A}_{f}} \exists_{d_{J} \in \mathbb{R}} \forall_{x \in J} g(x)=c f(x)+d_{J} .
$$

Using this form and Lemma 1 , for each $J \in \mathcal{A}_{f}$ we have the following cases:

(a) $\exists_{I_{1} \in \mathcal{A}_{f}}\left[\sup J, \inf I_{1}\right] \subset I \backslash U_{f}$.

Let $a=\sup J, b=\inf I_{1}$. We have

$$
\begin{aligned}
c f(a) & +d_{J}=\lim _{x \rightarrow a^{-}} c f(x)+d_{J}=\lim _{x \rightarrow a^{-}} g(x)=g(a)=g(b) \\
& =\lim _{x \rightarrow b^{+}} g(x)=\lim _{x \rightarrow b^{+}} c f(x)+d_{I_{1}}=c f(b)+d_{I_{1}}=c f(a)+d_{I_{1}} .
\end{aligned}
$$


Hence $d_{I_{1}}=d_{J}$.

(b) $\quad \exists_{\left(I_{n}\right)_{n \in \mathbb{N}} \subset \mathcal{A}_{f}}\left(\forall_{n \in \mathbb{N}} \inf I_{n+1} \geq \sup I_{n}\right) \wedge\left[\lim _{n \rightarrow \infty} \sup I_{n}, \inf J\right] \subset I \backslash U_{f}$.

Let $a=\lim _{n \rightarrow \infty} \sup I_{n}, b=\inf J$. In view of the previous case we get $d_{I_{n}}=d_{I_{n+1}}$. We have

$$
\begin{gathered}
c f(a)+d_{I_{n}}=\lim _{x \rightarrow a^{-}} c f(x)+d_{I_{n}}=\lim _{x \rightarrow a^{-}} g(x)=g(a)=g(b)=\lim _{x \rightarrow b^{+}} g(x) \\
=\lim _{x \rightarrow b^{+}} c f(x)+d_{J}=c f(b)+d_{J}=c f(a)+d_{J}, n \in \mathbb{N} .
\end{gathered}
$$

Hence $d_{I_{n}}=d_{J}$ for all $n \in \mathbb{N}$.

(c) $\exists_{I_{1} \in \mathcal{A}_{f}}\left[\sup I_{1}, \inf J\right] \subset I \backslash U_{f}$.

Let $a=\sup I_{1}, b=\inf J$. We have

$$
\begin{aligned}
c f(a) & +d_{I_{1}}=\lim _{x \rightarrow a^{-}} c f(x)+d_{I_{1}}=\lim _{x \rightarrow a^{-}} g(x)=g(a)=g(b) \\
& =\lim _{x \rightarrow b^{+}} g(x)=\lim _{x \rightarrow b^{+}} c f(x)+d_{J}=c f(b)+d_{J}=c f(a)+d_{J} .
\end{aligned}
$$

Hence $d_{I_{1}}=d_{J}$.

(d) $\exists_{\left(I_{n}\right)_{n \in \mathbb{N}} \subset \mathcal{A}_{f}}\left(\forall_{n \in \mathbb{N}} \sup I_{n+1} \leq \inf I_{n}\right) \wedge\left[\sup J, \lim _{n \rightarrow \infty} \inf I_{n}\right] \subset I \backslash U_{f}$.

Let $a=\sup J, b=\lim _{n \rightarrow \infty} \inf I_{n}$. In view of the previous case we get $d_{I_{n}}=d_{I_{n+1}}$. We have

$$
\begin{gathered}
c f(a)+d_{J}=\lim _{x \rightarrow a^{-}} c f(x)+d_{J}=\lim _{x \rightarrow a^{-}} g(x)=g(a)=g(b)=\lim _{x \rightarrow b^{+}} g(x) \\
=\lim _{x \rightarrow b^{+}} c f(x)+d_{I_{n}}=c f(b)+d_{I_{n}}=c f(a)+d_{I_{n}}, n \in \mathbb{N} .
\end{gathered}
$$

Hence $d_{I_{n}}=d_{J}$ for all $n \in \mathbb{N}$.

From the above we obtain that there exist $c, d \in \mathbb{R}$ such that $g(x)=c f(x)+d$ for all $x \in U_{f}$.

Now we will show that $g$ also has this form on $I \backslash U_{f}$. We have the following cases:

- Let $J \subset\left(\inf U_{f}, \sup U_{f}\right) \backslash U_{f}$ be a closed interval such that $a, b \in \operatorname{cl} U_{f}$, where $a=\inf J, b=\sup J$. Then we have

$$
\begin{aligned}
g(x) & =g(a)=\lim _{y \rightarrow a^{-}, y \in U_{f}} g(y)=\lim _{y \rightarrow a^{-}, y \in U_{f}} c f(x)+d \\
& =c f(a)+d=c f(x)+d, x \in J .
\end{aligned}
$$

- Assume that $a=\sup U_{f}<\infty$ and let $J=\left[\sup U_{f},+\infty\right)$. Then we have

$$
\begin{aligned}
g(x) & =g(a)=\lim _{y \rightarrow a^{-}, y \in U_{f}} g(y)=\lim _{y \rightarrow a^{-}, y \in U_{f}} c f(x)+d \\
& =c f(a)+d=c f(x)+d, x \in[a,+\infty) .
\end{aligned}
$$

- Assume that $a:=\inf I<b:=\inf U_{f}$. Let $x \in(a, b], z \in U_{f}, y:=\frac{z-\alpha x}{1-\alpha}$. Then $z=\alpha x+(1-\alpha) y$ and from the form of $g$ on $U_{f}$ and the above two 
cases we have $g(y)=c f(y)+d$. Hence

$$
\begin{aligned}
g(x) f^{\prime}(z)= & {[g(x)-g(y)] f^{\prime}(z)+g(y) f^{\prime}(z)=[f(x)-f(y)] g^{\prime}(z) } \\
& +[c f(y)+d] f^{\prime}(z)=[f(x)-f(y)] c f^{\prime}(z)+[c f(y)+d] f^{\prime}(z) \\
= & {[c f(x)+d] f^{\prime}(z), }
\end{aligned}
$$

which means that $g(x)=c f(x)+d$.

Assume that $f$ is non-constant and $\inf I=-\infty$. Let $F, G:-I \rightarrow \mathbb{R}$ be given by the formulas $F(x)=f(-x), G(x)=g(-x)$ for $x \in-I$. Then we have $F^{\prime}(x)=-f^{\prime}(-x), G^{\prime}(x)=-g^{\prime}(x)$ for $x \in-I$ and $F, G$ satisfy Eq. (1). Since $\sup -I=+\infty$, there exist $c, d \in \mathbb{R}$ such that $G(x)=c F(x)+d$ for $x \in-I$, which means that $g(x)=c f(x)+d$ for $x \in I$.

\section{Main result for the asymmetric case}

First, we consider the case when $\alpha \neq \frac{1}{2}$. We start with the following.

Lemma 3. Let $J \subset \mathbb{R}$ be an open interval, $f, g, h: J \rightarrow \mathbb{R}$ be continuous functions, $f$ be strictly monotone and the following functional equation hold

$$
g(x)-g(y)=h(\alpha x+(1-\alpha) y)[f(x)-f(y)], x, y \in J .
$$

Then there exist $c, d \in \mathbb{R}$ such that $g(x)=c f(x)+d, h(x)=c$ for all $x \in J$.

Proof. Let $u \in J$. We define two numbers

$$
\begin{aligned}
& a_{u}:=\max \left\{\frac{1-2 \alpha}{1-\alpha} \inf J+\frac{\alpha}{1-\alpha} u, \frac{1-\alpha}{\alpha} u-\frac{1-2 \alpha}{\alpha} \sup J\right\}, \\
& b_{u}:=\min \left\{\frac{1-2 \alpha}{1-\alpha} \sup J+\frac{\alpha}{1-\alpha} u, \frac{1-\alpha}{\alpha} u-\frac{1-2 \alpha}{\alpha} \inf J\right\} .
\end{aligned}
$$

We observe that

$$
\begin{aligned}
& \frac{1-2 \alpha}{1-\alpha} \inf J+\frac{\alpha}{1-\alpha} u<\frac{1-2 \alpha}{1-\alpha} u+\frac{\alpha}{1-\alpha} u=u, \\
& \frac{1-\alpha}{\alpha} u-\frac{1-2 \alpha}{\alpha} \sup J<\frac{1-\alpha}{\alpha} u-\frac{1-2 \alpha}{\alpha} u=u, \\
& \frac{1-2 \alpha}{1-\alpha} \sup J+\frac{\alpha}{1-\alpha} u>\frac{1-2 \alpha}{1-\alpha} u+\frac{\alpha}{1-\alpha} u=u, \\
& \frac{1-\alpha}{\alpha} u-\frac{1-2 \alpha}{\alpha} \inf J>\frac{1-\alpha}{\alpha} u-\frac{1-2 \alpha}{\alpha} u=u .
\end{aligned}
$$

Hence $a_{u}<u<b_{u}$. We define an open interval $I_{u}:=\left(a_{u}, b_{u}\right) \cap J$. Therefore $u \in I_{u}$. 
Let $v \in I_{u}$. Then for $x=\frac{1-\alpha}{1-2 \alpha} v-\frac{\alpha}{1-2 \alpha} u, y=\frac{1-\alpha}{1-2 \alpha} u-\frac{\alpha}{1-2 \alpha} v$ we have

$$
\begin{aligned}
& \alpha x+(1-\alpha) y=\frac{\left(\alpha-\alpha^{2}\right) v-\alpha^{2} u+\left(1-2 \alpha+\alpha^{2}\right) u-\left(\alpha-\alpha^{2}\right) v}{1-2 \alpha}=u, \\
& \alpha y+(1-\alpha) x=\frac{\left(\alpha-\alpha^{2}\right) u-\alpha^{2} v+\left(1-2 \alpha+\alpha^{2}\right) v-\left(\alpha-\alpha^{2}\right) u}{1-2 \alpha}=v .
\end{aligned}
$$

We also have

$$
\begin{aligned}
x= & \frac{1-\alpha}{1-2 \alpha} v-\frac{\alpha}{1-2 \alpha} u>\frac{(1-\alpha) a_{u}-\alpha u}{1-2 \alpha} \\
& \geq \frac{(1-2 \alpha) \inf J+\alpha u-\alpha u}{1-2 \alpha}=\inf J \\
x= & \frac{1-\alpha}{1-2 \alpha} v-\frac{\alpha}{1-2 \alpha} u<\frac{(1-\alpha) b_{u}-\alpha u}{1-2 \alpha} \\
& \leq \frac{(1-2 \alpha) \sup J+\alpha u-\alpha u}{1-2 \alpha}=\sup J \\
y= & \frac{1-\alpha}{1-2 \alpha} u-\frac{\alpha}{1-2 \alpha} v<\frac{(1-\alpha) u-\alpha a_{u}}{1-2 \alpha} \\
& \leq \frac{(1-\alpha) u-(1-\alpha) u+(1-2 \alpha) \sup J}{1-2 \alpha}=\sup J \\
y= & \frac{1-\alpha}{1-2 \alpha} u-\frac{\alpha}{1-2 \alpha} v>\frac{(1-\alpha) u-\alpha b_{u}}{1-2 \alpha} \\
& \geq \frac{(1-\alpha) u-(1-\alpha) u+(1-2 \alpha) \inf J}{1-2 \alpha}=\inf J
\end{aligned}
$$

which means that $x, y \in J$.

We observe that

$$
\begin{aligned}
h(u) & =h(\alpha x+(1-\alpha) y)=\frac{g(x)-g(y)}{f(x)-f(y)} \\
& =\frac{g(y)-g(x)}{f(y)-f(x)}=h(\alpha y+(1-\alpha) x)=h(v) .
\end{aligned}
$$

Hence we have that $h$ is constant on $I_{u}$.

Now we show that $h$ is constant. Fix $u, v \in J, u<v$. Let $c_{0}=u, c_{n}=\sup I_{c_{n-1}}$ for $n \in \mathbb{N}$. Since $c_{n-1} \in I_{c_{n-1}},\left(c_{n}\right)_{n \in \mathbb{N}}$ is strictly increasing. We also have $I_{c_{n-1}} \cap I_{c_{n}} \neq \emptyset$, so $h$ is constant on (inf $I_{u}, \lim _{n \rightarrow \infty} c_{n}$ ). If $\lim _{n \rightarrow \infty} c_{n}=+\infty$, then we get $h(u)=h(v)$. Assume that $\lim _{n \rightarrow \infty} c_{n}<+\infty$. We have

$$
\begin{aligned}
c_{n+1}-c_{n} & \geq \min \left\{\frac{1-2 \alpha}{1-\alpha} \sup J+\frac{\alpha}{1-\alpha} c_{n}, \frac{1-\alpha}{\alpha} c_{n}-\frac{1-2 \alpha}{\alpha} \inf J\right\}-c_{n} \\
& =\min \left\{\frac{1-2 \alpha}{1-\alpha}\left(\sup J-c_{n}\right), \frac{1-2 \alpha}{\alpha}\left(c_{n}-\inf J\right)\right\}, n \in \mathbb{N} .
\end{aligned}
$$


Since $c_{n}-\inf J>c_{1}-\inf J>0$ for $n \in \mathbb{N}$,

$$
0=\lim _{n \rightarrow \infty}\left(c_{n+1}-c_{n}\right)=\lim _{n \rightarrow \infty} \frac{1-2 \alpha}{1-\alpha}\left(\sup J-c_{n}\right),
$$

which means that $\lim _{n \rightarrow \infty} c_{n}=\sup J$ and we get $h(u)=h(v)$.

Let $c:=h(u)$ for $u \in J$. Fix $y \in J$ and let $d:=g(y)-c f(y)$. We observe that

$$
\begin{aligned}
g(x) & =g(x)-g(y)+g(y)=[f(x)-f(y)] h(\alpha x+(1-\alpha) y)+g(y) \\
& =c f(x)-c f(y)+g(y)=c f(x)+d, x \in J
\end{aligned}
$$

which ends the proof.

Corollary 4. Let $J \subset \mathbb{R}$ be an open interval, $f, g: J \rightarrow \mathbb{R}$ be differentiable functions such that (1) holds and $f^{\prime}(x) \neq 0$ for all $x \in J$. Then there exist $c, d \in \mathbb{R}$ such that $g(x)=c f(x)+d$ for all $x \in J$.

In view of the above corollary we obtain

Corollary 5. Let $f, g: I \rightarrow \mathbb{R}$ be differentiable and satisfy Eq. (1). Then $\{1, f, g\}$ are linearly dependent on each $J \in \mathcal{A}_{f}$.

Finally we have the main result for the asymmetric case.

Theorem 6. Let $f, g: I \rightarrow \mathbb{R}$ be differentiable and satisfy Eq. (1). Assume that $\inf I=-\infty$ or $\sup I=+\infty$. Then either $f$ is constant and $g$ is an arbitrary function or there exist $c, d \in \mathbb{R}$ such that $g(x)=c f(x)+d$ for $x \in I$.

Proof. In view of Corollary 5 and Lemma 2 we obtain the thesis of this theorem.

Remark 7. It is easy to see that for differentiable functions $f, g: I \rightarrow \mathbb{R}$, if $\{1, f, g\}$ are linearly dependent, then (1) holds.

\section{Main result for the symmetric case}

Now we consider the case when $\alpha=\frac{1}{2}$.

Lundberg in his papers [4, Table 1], [5, Theorem 1.2] considers the following functional equation

$$
\varphi(x+y)=\frac{F(x) G(y)+H(x) L(y)}{m(x)+n(y)}
$$

on rectangles in $\mathbb{R}^{2}$ for continuous functions $F, G, H, L, m, n, \varphi$, which is a generalization of Eq. (1) on each $J \in \mathcal{A}_{f}$. He presents solutions of this equation but they have indirect forms, so we use only the fact that for differentiable functions $f, g: I \rightarrow \mathbb{R}$ which satisfy Eq. (1) we have two cases on every open interval $J \in \mathcal{A}_{f}$ : either $\{1, f, g\}$ are linearly dependent on $J$ or $f$ and $g$ are 
infinitely differentiable. Particularly, on every $J \in \mathcal{A}_{f}$, if $\{1, f, g\}$ are linearly independent, then they are three times differentiable and we can use the following two facts from [2].

Remark 8 (see [2, Remark 10]). Let $f, g: I \rightarrow \mathbb{R}$ be differentiable functions which satisfy (1). On every interval $J \in \mathcal{A}_{f}$ one of the following cases holds:

(a) $\{1, f, g\}$ are linearly dependent;

(b) $f, g \in \operatorname{Lin}\left\{1, \mathrm{id}, \mathrm{id}^{2}\right\}$;

(c) $f, g \in \operatorname{Lin}\left\{1, e^{\mu \mathrm{id}}, e^{-\mu \mathrm{id}}\right\}$ for some $\mu>0$;

(d) $\quad f, g \in \operatorname{Lin}\{1, \sin (\mu \mathrm{id}), \cos (\mu \mathrm{id})\}$ for some $\mu>0$;

where id is the identity on $J$.

Lemma 9 (see [2, Lemma 11]). Let $f, g: I \rightarrow \mathbb{R}$ be differentiable functions which satisfy (1), $J \in \mathcal{A}_{f}$ be such that $g^{\prime}(a)=0$, where $a=\inf J>\inf I$ or $a=\sup J<\sup I$. Then $\{1, f, g\}$ are linearly dependent on $J$.

This lemma is proved in the case $a=\inf J>\inf I$, but the proof in the case $a=\sup J<\sup I$ is analogous.

Now we are ready to prove the main result.

Theorem 10. Let $f, g: I \rightarrow \mathbb{R}$ be differentiable and satisfy Eq. (1). Then one of the following possibilities holds:

(a) $\{1, f, g\}$ are linearly dependent on each $J \in \mathcal{A}_{f}$;

(b) $f, g \in \operatorname{Lin}\left\{1, i d, i d^{2}\right\}$;

(c) $f, g \in \operatorname{Lin}\left\{1, e^{\mu i d}, e^{-\mu i d}\right\}$ for some $\mu>0$;

(d) $f, g \in \operatorname{Lin}\{1, \sin (\mu i d), \cos (\mu i d)\}$ for some $\mu>0$.

Proof. We can split $\mathcal{A}_{f}$ into disjoint subsets $\mathcal{A}_{f}=\mathcal{L}_{f} \cup \mathcal{Q}_{f} \cup \mathcal{E}_{f} \cup \mathcal{T}_{f}$, where

$\mathcal{L}_{f}=\left\{J \in \mathcal{A}_{f}:\left\{1,\left.f\right|_{J},\left.g\right|_{J}\right\}\right.$ are linearly dependent $\}$,

$\mathcal{Q}_{f}=\left\{J \in \mathcal{A}_{f} \backslash \mathcal{L}_{f}:\left.f\right|_{J},\left.g\right|_{J} \in \operatorname{Lin}\left\{1, \mathrm{id}, \mathrm{id}^{2}\right\}\right\}$,

$\mathcal{E}_{f}=\left\{J \in \mathcal{A}_{f} \backslash \mathcal{L}_{f}:\left.f\right|_{J},\left.g\right|_{J} \in \operatorname{Lin}\left\{1, e^{\mu \mathrm{id}}, e^{-\mu \mathrm{id}}\right\}\right.$ for some $\left.\mu>0\right\}$,

$\mathcal{T}_{f}=\left\{J \in \mathcal{A}_{f} \backslash \mathcal{L}_{f}:\left.f\right|_{J},\left.g\right|_{J} \in \operatorname{Lin}\{1, \sin (\mu \mathrm{id}), \cos (\mu \mathrm{id})\}\right.$ for some $\left.\mu>0\right\}$.

We have the same split for $\mathcal{A}_{g}$.

If $U_{f}=U_{g}=I$, then in view of Remark 8 we have the thesis of this theorem, so we can assume that $U_{f} \neq I$.

Now we consider four cases:

- Assume that $\mathcal{Q}_{f} \neq \emptyset$. Let $I \in \mathcal{Q}_{f}$. We have $f^{\prime}(p)=0$, where $p=\inf J>$ $\inf I$ or $p=\sup J<\sup I$. Since there exist $a_{1}, a_{2}, a_{3}, b_{1}, b_{2}, b_{3} \in \mathbb{R}$ such that $f(x)=a_{1} x^{2}+a_{2} x+a_{3}, g(x)=b_{1} x^{2}+b_{2} x+b_{3}$ for $x \in J$ and $f^{\prime}$ on cl $J$ has only one root, we have that $0=f^{\prime}(p)=2 a_{1} p+a_{2}$ and $J=(p, \sup I)$ or $(\inf I, p)$. In view of Lemma 9 we have $g^{\prime}(p) \neq 0$. Hence

$$
\begin{aligned}
0= & {[g(p+h)-g(p-h)] f^{\prime}(p)=[f(p+h)-f(p-h)] g^{\prime}(p), } \\
& h \in \mathbb{R}, p+h, p-h \in I,
\end{aligned}
$$


which means that

$$
f(p+h)=f(p-h), h \in \mathbb{R}, p+h, p-h \in I .
$$

We have

$$
\begin{aligned}
f(p-h) & =f(p+h)=a_{1}(p+h)^{2}+a_{2}(p+h)+a_{3} \\
& =a_{1}(p-h)^{2}+a_{2}(p-h)+a_{3}+2 h\left(2 a_{1} p+a_{2}\right) \\
& =a_{1}(p-h)^{2}+a_{2}(p-h)+a_{3}, h \in \mathbb{R}, p+h \in J, p-h \in I,
\end{aligned}
$$

so the set $(2 p-J) \cap I$ is a subset of some $J_{2} \in \mathcal{Q}_{f}(\{1, f, g\}$ are linearly independent on $J$ so also on $(2 p-J) \cap I)$. Since $f^{\prime}$ on $\mathrm{cl} J_{2}$ has only one root $p, J_{2}=I \backslash J$. Hence $f(x)=a_{1} x^{2}+a_{2} x+a_{3}$ for $x \in I$ and we can also prove in a similar way that $g(x)=b_{1} x^{2}+b_{2} x+b_{3}$ for $x \in I$.

- Assume that $\mathcal{E}_{f} \neq \emptyset$. Let $I \in \mathcal{E}_{f}$. We have $f^{\prime}(p)=0$, where $p=\inf J>\inf I$ or $p=\sup J<\sup I$. Since there exist $\mu>0, a_{1}, a_{2}, a_{3}, b_{1}, b_{2}, b_{3} \in \mathbb{R}$ such that $f(x)=a_{1} e^{\mu x}+a_{2} e^{-\mu x}+a_{3}, g(x)=b_{1} e^{\mu x}+b_{2} e^{-\mu x}+b_{3}$ for $x \in J$ and $f^{\prime}$ on $\mathrm{cl} J$ has only one root, we have that $0=f^{\prime}(p)=\mu\left(a_{1} e^{\mu p}-a_{2} e^{-\mu p}\right)$ and $J=(p, \sup I)$ or (inf $I, p)$. Similarly as in the first case we obtain (5). We also have

$$
\begin{aligned}
f(p-h) & =f(p+h)=a_{1} e^{\mu(p+h)}+a_{2} e^{-\mu(p+h)}+a_{3} \\
& =a_{1} e^{\mu(p-h)}+a_{2} e^{-\mu(p-h)}+a_{3}+\left(e^{\mu h}-e^{-\mu h}\right)\left(a_{1} e^{\mu p}-a_{2} e^{-\mu p}\right) \\
& =a_{1} e^{\mu(p-h)}+a_{2} e^{-\mu(p-h)}+a_{3}, h \in \mathbb{R}, p+h \in J, p-h \in I,
\end{aligned}
$$

so the set $(2 p-J) \cap I$ is a subset of some $J_{2} \in \mathcal{E}_{f}(\{1, f, g\}$ are linearly independent on $J$ so also on $(2 p-J) \cap I)$. Since $f^{\prime}$ on $\mathrm{cl} J_{2}$ has only one root $p, J_{2}=I \backslash J$. Hence $f(x)=a_{1} e^{\mu x}+a_{2} e^{-\mu x}+a_{3}$ for $x \in I$ and we can also prove in a similar way that $g(x)=b_{1} e^{\mu x}+b_{2} e^{-\mu x}+b_{3}$ for $x \in I$.

- Assume that $\mathcal{T}_{f} \neq \emptyset$. Let $I \in \mathcal{T}_{f}$. We have $f^{\prime}(p)=0$, where $p=\inf J>\inf I$ or $p=\sup J<\sup I$. Since there exist $\mu>0, a_{1}, a_{2}, a_{3}, b_{1}, b_{2}, b_{3} \in \mathbb{R}$ such that $f(x)=a_{1} \cos (\mu x)+a_{2} \sin (\mu x)+a_{3}, g(x)=b_{1} \cos (\mu x)+b_{2} \sin (\mu x)+b_{3}$ for $x \in J$, we have that $0=f^{\prime}(p)=\mu\left(a_{2} \cos (\mu p)-a_{1} \sin (\mu p)\right)$. Similarly as in the first case we obtain (5). We also have

$$
\begin{aligned}
& f(p-h)=f(p+h)=a_{1} \cos (\mu(p+h))+a_{2} \sin (\mu(p+h))+a_{3} \\
& =a_{1} \cos (\mu(p-h))+a_{2} \sin (\mu(p-h))+a_{3} \\
& \quad+a_{1}[\cos (\mu(p+h))-\cos (\mu(p-h))]+a_{2}[\sin (\mu(p+h))-\sin (\mu(p-h))] \\
& =a_{1} \cos (\mu(p-h))+a_{2} \sin (\mu(p-h))+a_{3} \\
& \quad+2 a_{2} \sin (\mu p) \sin (-\mu h)+2 a_{1} \sin (\mu h) \cos (\mu p) \\
& =a_{1} \cos (\mu(p-h))+a_{2} \sin (\mu(p-h))+a_{3}
\end{aligned}
$$




$$
\begin{aligned}
& +2 \sin (\mu h)\left[a_{2} \cos (\mu p)-a_{1} \sin (\mu p)\right] \\
= & a_{1} \cos (\mu(p-h))+a_{2} \sin (\mu(p-h))+a_{3}, h \in \mathbb{R}, p+h \in J, p-h \in I,
\end{aligned}
$$

so the set $(2 p-J) \cap I$ is a subset of some $J_{2} \in \mathcal{T}_{f}(\{1, f, g\}$ are linearly independent on $J$ so also on $(2 p-J) \cap I)$. Using this method we obtain that $f(x)=a_{1} \cos (\mu x)+a_{2} \sin (\mu x)+a_{3}$ for $x \in I$ and we can also prove in a similar way that $g(x)=b_{1} e^{\mu x}+b_{2} e^{-\mu x}+b_{3}$ for $x \in I$.

- Assume that $\mathcal{L}_{f} \neq \emptyset$. From the three previous cases we get that $\mathcal{A}_{f}=\mathcal{L}_{f}$.

Theorem 11. Let $f, g: I \rightarrow \mathbb{R}$ be differentiable and satisfy Eq. (1) and $\sup I=$ $+\infty$ or $\inf I=-\infty$. Then one of the following possibilities holds:

(a) $\{1, f, g\}$ are linearly dependent;

(b) $f, g \in \operatorname{Lin}\left\{1, i d, i d^{2}\right\}$;

(c) $f, g \in \operatorname{Lin}\left\{1, e^{\mu i d}, e^{-\mu i d}\right\}$ for some $\mu>0$;

(d) $f, g \in \operatorname{Lin}\{1, \sin (\mu i d), \cos (\mu i d)\}$ for some $\mu>0$.

Proof. We have only to show that in the first case we have the linear dependence on the whole $I$, which follows from Lemma 2 .

Remark 12. It is not difficult to check that (1) holds for differentiable functions $f, g: I \rightarrow \mathbb{R}$ which have one of the forms a - d on (arbitrary) $I$.

\section{Final remarks}

If we have an arbitrary open interval we cannot obtain the linear dependency of $\{1, f, g\}$ on the whole $I$ as in Theorems 6 and 11 . We have some arbitrariness near the ends of the interval (Example 13) or different coefficients of linear dependence between $f$ and $g$ on the intervals from $\mathcal{A}_{f}$ (Example 14).

Example 13. Let $h:(-1, \alpha-1) \rightarrow \mathbb{R}$ be a differentiable function such that $\lim _{x \rightarrow(\alpha-1)^{-}} h(x)=1$ and $\lim _{x \rightarrow(\alpha-1)^{-}} h^{\prime}(x)=0$, functions $f, g:(-1, \alpha) \rightarrow \mathbb{R}$ are given by the formulas

$$
\begin{aligned}
& f(x)= \begin{cases}0, & x \in(-1,0] \\
x^{2}, & x \in(0, \alpha)\end{cases} \\
& g(x)= \begin{cases}h(x), & x \in(-1, \alpha-1) \\
1, & x \in[\alpha-1,0] \\
x^{2}+1, & x \in(0, \alpha)\end{cases}
\end{aligned}
$$

Then $f$ and $g$ satisfy Eq. (1). Indeed, we have

- if $x, y \geq \alpha-1$, then $\alpha x+(1-\alpha) y \geq \alpha-1$ and since $g(z)=f(z)+1$ for $z \geq \alpha-1$, we get $f^{\prime}(\alpha x+(1-\alpha) y)=g^{\prime}(\alpha x+(1-\alpha) y)$ and $f(x)-f(y)=$ $g(x)-g(y)$. 
- if $x, y \leq 0$ then $\alpha x+(1-\alpha) y \leq 0$, so we get $f^{\prime}(\alpha x+(1-\alpha) y)=0$ and $f(x)-f(y)=0$.

- if $x<\alpha-1$ and $y>0$, then

$$
\begin{aligned}
& \alpha x+(1-\alpha) y>\alpha x>-\alpha \geq \alpha-1, \\
& \alpha x+(1-\alpha) y<\alpha(\alpha-1)+(1-\alpha) \alpha=0,
\end{aligned}
$$

so $\alpha x+(1-\alpha) y \in(\alpha-1,0)$, which give us $f^{\prime}(\alpha x+(1-\alpha) y)=0$ and $g^{\prime}(\alpha x+(1-\alpha) y)=0$.

- if $y<\alpha-1$ and $x>0$, then

$$
\begin{aligned}
& \alpha x+(1-\alpha) y>(1-\alpha) y>1-\alpha, \\
& \alpha x+(1-\alpha) y<\alpha \alpha+(1-\alpha)(\alpha-1)=2 \alpha-1 \leq 0,
\end{aligned}
$$

so $\alpha x+(1-\alpha) y \in(\alpha-1,0)$, which give us $f^{\prime}(\alpha x+(1-\alpha) y)=0$ and $g^{\prime}(\alpha x+(1-\alpha) y)=0$.

Example 14. Let $c \in \mathbb{R}$, functions $f, g:\left(-\frac{\alpha}{1-\alpha}, \frac{1}{1-\alpha}\right) \rightarrow \mathbb{R}$ be given by the formulas

$$
\begin{aligned}
& f(x)=\left\{\begin{array}{ll}
-x^{2}, & x \in\left(-\frac{\alpha}{1-\alpha}, 0\right) \\
0, & x \in[0,1] \\
(x-1)^{2}, & x \in\left(1, \frac{1}{1-\alpha}\right)
\end{array},\right. \\
& g(x)=\left\{\begin{array}{ll}
-c x^{2}, & x \in\left(-\frac{\alpha}{1-\alpha}, 0\right) \\
0, & x \in[0,1] \\
(x-1)^{2}, & x \in\left(1, \frac{1}{1-\alpha}\right)
\end{array} .\right.
\end{aligned}
$$

Then $f$ and $g$ satisfy Eq. (1). Indeed, we have

- if $x, y \in\left(-\frac{\alpha}{1-\alpha}, 0\right)$ or $x, y \in[0,1]$ or $x, y \in\left(1, \frac{1}{1-\alpha}\right)$, then it is obvious.

- if $x<0$ and $y>1$, then

$$
0 \leq 1-2 \alpha \leq-\alpha+(1-\alpha) \leq \alpha x+(1-\alpha) y \leq(1-\alpha) y \leq 1,
$$

so $f^{\prime}(\alpha x+(1-\alpha) y)=g^{\prime}(\alpha x+(1-\alpha) y)=0$.

- if $y<0$ and $x>1$, then

$$
0=\alpha-(1-\alpha) \frac{\alpha}{1-\alpha} \leq \alpha x+(1-\alpha) y \leq \alpha x \leq \frac{\alpha}{1-\alpha} \leq 1,
$$

so $f^{\prime}(\alpha x+(1-\alpha) y)=g^{\prime}(\alpha x+(1-\alpha) y)=0$.

- if $x<0 \leq y \leq 1$ then either $\alpha x+(1-\alpha) y \in[0,1]$ (and we get $f^{\prime}(\alpha x+$ $\left.(1-\alpha) y)=g^{\prime}(\alpha x+(1-\alpha) y)=0\right)$ or $\alpha x+(1-\alpha) y<0$ (and we obtain $\left.f(x) g^{\prime}(\alpha x+(1-\alpha) y)=g(x) f^{\prime}(\alpha x+(1-\alpha) y)\right)$.

Analogously we have the case when $y<0 \leq x \leq 1$.

- if $0 \leq x \leq 1<y$ then either $\alpha x+(1-\alpha) y \in[0,1]$ (and we get $f^{\prime}(\alpha x+$ $\left.(1-\alpha) y)=g^{\prime}(\alpha x+(1-\alpha) y)=0\right)$ or $\alpha x+(1-\alpha) y>1$ (and we obtain $\left.f(x) g^{\prime}(\alpha x+(1-\alpha) y)=g(x) f^{\prime}(\alpha x+(1-\alpha) y)\right)$.

Analogously we have the case when $0 \leq y \leq 1<x$. 
Sahoo and Riedel [6, 9, Sect. 2.7] posed the following

Problem 15. Find all functions $f, g, \varphi, \psi: \mathbb{R} \rightarrow \mathbb{R}$ satisfying

$$
[f(x)-f(y)] \varphi\left(\frac{x+y}{2}\right)=[g(x)-g(y)] \psi\left(\frac{x+y}{2}\right), x, y \in \mathbb{R} .
$$

This problem was solved by Balogh et al. [2, Theorem 12] for three times differentiable functions $f, g$.

Using Theorem 11 we can extend their solutions for differentiable functions (the proof is similar).

Theorem 16. Let $f, g: I \rightarrow \mathbb{R}$ be differentiable and $\varphi, \psi: I \rightarrow \mathbb{R}$ be arbitrary functions satisfying $E q$. (6), $\sup I=+\infty$ or $\inf I=-\infty$. If $\forall_{x \in I}[\varphi(x) \neq$ $0 \vee \psi(x) \neq 0]$, then one of the following possibilities holds:

(a) there exist constants $A_{0}, A_{1}, A_{2} \in \mathbb{R}$ such that for all $s \in I$ we have $A_{0}+A_{1} f(s)+A_{2} g(s)=0$ and $\left[A_{1} \psi(s)+A_{2} \varphi(s)\right] g^{\prime}(s)=0 ;$

(b) there exist constants $A_{0}, A_{1}, A_{2}, B_{0}, B_{1}, B_{2} \in \mathbb{R}$ such that for all $s \in I$ we have $f(s)=A_{0}+A_{1} s+A_{2} s^{2}, g(s)=B_{0}+B_{1} s+B_{2} s^{2}$ and

$$
\left[A_{1}+2 A_{2} s\right] \varphi(s)=\left[B_{1}+2 B_{2} s\right] \psi(s)
$$

(c) there exist $\mu>0$ and constants $A_{0}, A_{1}, A_{2}, B_{0}, B_{1}, B_{2} \in \mathbb{R}$ such that for all $s \in I$ we have $f(s)=A_{0}+A_{1} e^{\mu s}+A_{2} e^{-\mu s}, g(s)=B_{0}+B_{1} e^{\mu s}+$ $B_{2} e^{-\mu s}$ and

$$
\left[A_{1} e^{\mu s}-A_{2} e^{-\mu s}\right] \varphi(s)=\left[B_{1} e^{\mu s}-B_{2} e^{-\mu s}\right] \psi(s) ;
$$

(d) there exist $\mu>0$ and constants $A_{0}, A_{1}, A_{2}, B_{0}, B_{1}, B_{2} \in \mathbb{R}$ such that for all $s \in I$ we have $f(s)=A_{0}+A_{1} \sin (\mu s)+A_{2} \cos (\mu s), g(s)=$ $B_{0}+B_{1} \sin (\mu s)+B_{2} \cos (\mu s)$ and

$$
\left[A_{1} \cos (\mu s)-A_{2} \sin (\mu s)\right] \varphi(s)=\left[B_{1} \cos (\mu s)-B_{2} \sin (\mu s)\right] \psi(s) \text {. }
$$

Open Access. This article is distributed under the terms of the Creative Commons Attribution 4.0 International License (http://creativecommons.org/licenses/by/4.0/), which permits unrestricted use, distribution, and reproduction in any medium, provided you give appropriate credit to the original author(s) and the source, provide a link to the Creative Commons license, and indicate if changes were made.

\section{References}

[1] Aczél, J.: A mean value property of the derivative of quadratic polynomials-without mean values and derivatives. Math. Mag. 58(1), 42-45 (1985)

[2] Balogh, Z.M., Ibrogimov, O.O., Mityagin, B.S.: Functional equations and the Cauchy mean value theorem. Aequ. Math. 90, 683-697 (2016)

[3] Haruki, S.: A property of quadratic polynomials. Am. Math. Monthly 86(7), 577-579 (1979)

[4] Lundberg, A.: A rational Sûto equation. Aequ. Math. 57, 254-277 (1999) 
[5] Lundberg, A.: Sequential derivatives and their application to a Sûto equation. Aequ. Math. 61, 48-59 (2001)

[6] Sahoo, P.K., Riedel, T.: Mean Value Theorems and Functional Equations. World Scientific Publishing Co., Inc., River Edge (1998)

Radosław Łukasik

Institute of Mathematics

University of Silesia

ul. Bankowa 14

40-007 Katowice

Poland

e-mail: rlukasik@math.us.edu.pl

Received: August 28, 2017

Revised: May 24, 2018 